\title{
Association between telomere length in the DNA of peripheral blood leukocytes and the propofol dose in anesthesia induction
}

\author{
JunLong Zhang
}

First Affiliated Lianyungang No. 2 Poeple's Hospital of Jiangsu University

\section{Yan Xu}

the 74th Group Army Hospital of P.L.A

Chuangbo Xie

The Southern Theater General Hospital

Jing Yang

the southern theater general hospital

YongJi Xing

The 74th Group Army Hospital of P.L.A.

\section{WenBin Xi}

The Southern Theater General Hospital

\section{ZhaoJu Li}

The Southern Theater General Hospital

weifeng tu (D18928380889@163.com)

The Southern Theater General Hospital

\section{Research article}

Keywords: Telomere length, Propofol dose, Improve the anesthesia safety, Reduce perioperative complications

Posted Date: March 18th, 2020

DOI: https://doi.org/10.21203/rs.3.rs-17808/v1

License: (c) (i) This work is licensed under a Creative Commons Attribution 4.0 International License. Read Full License 


\section{Abstract}

Background Propofol is a widely used anesthetic and its dose is closely related to aging. Telomere length $(T L)$ is a unique heritable trait, and emerging as a biomarker of aging, health and disease. We proposed a hypothesis that propofol dose can be determined by telomere length, which greatly reduced the risk of anesthesia, especially the elderly.

Methods/design To evaluate the association between the propofol dose in anesthesia induction and: TL in the DNA of peripheral blood leukocytes; body weight;sex;difference of the Bispectral Index (BIS) before and after anesthesia induction in patients. To detect deletion at the 5'end or 3 'end of telomerase RNA component (TERC). 100 patients scheduled for elective surgery. Multivariable linear regression analyses were undertaken.

Results We found that propofol dose in anesthesia induction was clearly correlated significantly with TL $(r=0.78, p<0.001)$, sex $(r=0.83, p<0.001)$, body weight $(r=0.84, p=0.004)$, and difference of BIS before and after anesthesia induction $(r=0.85, p=0.029)$. By comparing the absolute values of standardized regression coefficients $(0.58,0.19,0.21$, and 0.12$)$ of the four variables, it can be seen that TL contributes the most to the propofol dose in anesthesia induction. However, detection of deletion at the $5^{\prime}$ end or $3^{\prime}$ end of TERC was not found, which showed that telomerase in these patients was relatively stable.

Discussion These findings provide preliminary evidence that the propofol dose in anesthesia induction was clearly correlated with genetically determined TL. TL may be a promising predictor of the propofol dose, which is beneficial to improve the safety of anesthesia and reduce perioperative complications.

\section{Background}

Ageing populations are becoming increasingly serious social problems. The number of elderly people who need surgery and anesthesia due to illness is also increasing. Furthermore, the elderly constitutes the most important population within the general population with regard to complications and deaths from anesthesia and surgery[1].

Evidence-based medical data have shown that perioperative complications are due mainly to an overdose of the drugs used to induce general anesthesia, hemodynamic disorders, and poor recovery from anesthesia[2]. Tolerance to intravenous general anesthesia decreases gradually with age in humans[3]. However, the extent of physiological changes within the body of individuals varies as they get older.

To date, there are no precise criteria for assessing the change in tolerance of the body to anesthetic drugs. The anesthesiologist, in general, adjusts the dose of anesthetic drugs in patients using the "actual age" (i.e., the chronological age based on time and year) combined with disease status, and personal clinical experience to reduce the risk of anesthesia and the surgical procedure. There are obvious shortcomings to this strategy. 
Telomeres are specialized protein-bound DNA repetitive sequences at the end of eukaryotic chromosomes. They regulate the replication and proliferation of cells, avoid chromosome fusion during mitosis, and maintain genomic stability[4]. Telomere length (TL) is a unique heritable trait, and has emerged as a biomarker of aging, health and disease. TL in leukocytes shortens in a divinable way with age by approximately 20-40 base pairs (bp) per year[5]. The aging and subsequent death of cells often happens if the mean TL reaches a critical value[6].

Hence, we proposed a hypothesis that propofol dose can be determined by telomere length. We designed a study to evaluate the association between TL in the DNA of peripheral blood leukocytes (PBLs) and propofol dose in the induction of general anesthesia.

\section{Methods}

\section{Ethical considerations}

This study was approved by Ethics Committee of Guangzhou General Hospital of Guangzhou Military Command and written informed consent was obtained from all participants prior to in the trial. All participants' rights will be protected. The trial was registered prior to patient enrollment at clinicaltrials.gov (NCT03429309, Principal investigator: WeiFeng Tu, Date of registration: February 9, 2018)

\section{Participants}

Recruiting participants began on 1 April 2018, and completed on 1 October 2018 in this study. After exclusion of 5 patients, one-hundred patients aged from 18 years to 80 years, with American Society of Anesthesiologists physical status I-II, were included in the study.

\section{Aims and design}

To evaluate the association between the propofol dose in anesthesia induction and: TL in the DNA of peripheral blood leukocytes; body weight; sex;difference of the Bispectral Index (BIS) before and after anesthesia induction in patients. To detect deletion at the 5 'end or 3 'end of telomerase RNA component (TERC). Patients were scheduled for elective surgery after arriving in the operating theatre, a peripheral venous catheter was inserted for infusion of fluids and drugs. The heart rate, peripheral oxygen saturation, noninvasive blood pressure and BIS were monitored continuously. Also, 100\% oxygen was given for 3 min by face mask. The induction of anesthesia was started by infusion of propofol (Fresenius Kabi, Bad Homburg vor der Höhe, Germany) using an intravenous syringe pump (B. Braun Melsungen, Germany) at $30 \mathrm{mg} / \mathrm{kg} / \mathrm{h}$. Recording began before propofol infusion was started.

When patients had a BIS of 70, they were asked loudly to "open their eyes". This command was repeated up to three times, and the eyelash reflex was examined at 15-s intervals until they became unconscious (i.e., lost response to a verbal command, no spontaneous movements and loss of the eyelash reflex) by the anesthetist. Recording of the heart rate, noninvasive blood pressure, and BIS was done when patients 
were awake (baseline) until propofol induced unconsciousness. The propofol dose and time taken for consciousness to disappear were recorded after propofol administration. Patients were instructed not to open their eyes, talk, or move during recording of the heart rate, noninvasive blood pressure, and BIS before propofol infusion. The sedation level was assessed until the patient became unconscious. Research index of patients (Table 1).

Table 1

Research index of patients

\begin{tabular}{|ll|}
\hline Research index & Characteristics \\
\hline patients & $\mathrm{N}=100$ \\
\hline Age (years) & $18-80$ \\
\hline Propofol dose in anesthesia induction & $30 \mathrm{mg} / \mathrm{kg} / \mathrm{h} /$ \\
\hline Telomere length & In PBLs \\
\hline Detection of deletion at the 5' or 3'end of TERC & \\
\hline Difference of BIS before and after anesthesia induction & \\
\hline Body weight (kg) & $40-87$ \\
\hline BMI & $30 \mathrm{~kg} / \mathrm{m}^{2}>\mathrm{BMI}>18 \mathrm{~kg} / \mathrm{m}^{2}$ \\
\hline Sex(female, male) & $\mathrm{female}=44, \mathrm{male}=46$ \\
\hline
\end{tabular}

\section{Exclusion criteria}

We excluded people: with known cardiac, hepatic, pulmonary or renal disease; hearing disorders, neurologic diseases or diabetes mellitus; consuming $>20 \mathrm{~g}$ of alcohol daily; with a body mass index $\left(18 \mathrm{~kg} / \mathrm{m}^{2}>\mathrm{BMl}>30 \mathrm{~kg} / \mathrm{m}^{2}\right)$.

\section{TL measurement}

Blood samples were obtained from 100 patients during surgery. TL of DNA in PBLs pre-treatment was assessed by quantitative reverse-transcription-polymerase chain reaction (qRT-PCR), as described previously[7, 8]. Total PBLs were separated using Red Blood Cell Lysis Buffer (C3702; Beyotime Institute of Biotechnology, Beijing, China). Genomic DNA was extracted from PBLs using a DNA Blood kit (Shanghai Majorbio Pharmaceutical Technology, Shanghai, China). qRT-PCR was done in a 7500 Real Time PCR system (Applied Biosystems, Foster City, CA). The relative ratio of telomere-repeat copy number to single-copy number (T/S ratio) was calculated. Samples were compared with a reference DNA sample.

\section{Detection of deletion at the 5 'end or 3'end of telomerase RNA component (TERC)}

TERC provides instructions for making one component of the enzyme telomerase. 
qRT-PCR was used to detect gene deletion at the 5'end or 3'end of TERC, as described previously[9]. The reference gene $\beta$-globin was used for calculation of the copy number of TERC. An amplicon of size $1190 \mathrm{bp}$ extending from $433 \mathrm{bp}$ at the 5 'end flanking to 306-bp downstream of TERC was amplified for TERC sequencing. Genomic DNA was obtained with a SYBR ${ }^{\text {TM }}$ Premix Ex Taq kit (AK6006; TaKaRa Biotechnology, Shiga, Japan). The individual copy number of TERC at the 5 'end or 3 'end was calculated as the ratio of TERC/ $\beta$-globin of each sample using the comparative CT method $\left(2^{-\triangle \Delta C T}\right)$ [9].

\section{Statistical analysis}

Multivariable linear regression analyses were undertaken to assess the relationship between the: propofol dose in anesthesia induction; TL; Body weight; sex and difference of BIS before and after anesthesia induction, $p<0.05$ was considered significant. Analyses were done using SPSS v21.0 (IBM, Armonk, NY, USA).

\section{Results}

\section{Association between the propofol dose and other factors}

The propofol dose in anesthesia induction was clearly correlated significantly with $\operatorname{TL}(r=0.78, p<0.001)$; sex $(r=0.83, p<0.001)$; body weight $(r=0.84, p=0.004)$; and difference of BIS before and after anesthesia induction $(r=0.85, p=0.029)$. By comparing the absolute values of standardized regression coefficients $(0.58,0.19,0.21$, and 0.12$)$ of the four variables, it can be seen that TL contributes the most to the propofol dose in anesthesia induction( Table 2).

Table 2 Statistical results of the relationship between five variables

$\left(\right.$ Coefficients $\left.^{\mathrm{a}}\right)$

\begin{tabular}{|llll|}
\hline \multirow{2}{*}{ Model } & \multicolumn{2}{l|}{ Standardized Coefficients } & \\
\cline { 2 - 3 } & Beta & t & Sig. \\
\hline (Constant) & & 2.450 & .016 \\
\hline Tel & .578 & 8.977 & .000 \\
\hline Sex & .189 & 2.953 & .004 \\
\hline Body Weight & .206 & 2.984 & .004 \\
\hline BIS & .124 & 2.215 & .029 \\
\hline
\end{tabular}

1. Dependent Variable: Propofol Dose 


\section{Linear correlation scatter / dot between the propofol dose and other factors}

The propofol dose in anesthesia induction was positively correlated with TL; body weight; difference of BIS before and after anesthesia induction from the scatter diagram (Fig. 1).

\section{Detection of deletion at the $5^{\prime}$ end or 3 'end of TERC}

Detection of deletion at the 5 'end or 3 'end of TERC was not found in our 100 study participants. This result showed that telomerase in these patients was relatively stable.

\section{Discussion}

We found a positive association between TL in DNA in PBLs and the propofol dose in anesthesia induction (Table 2). Also, the propofol dose decreased with shorter TL (Fig. 1). Our study could reveal an association between the propofol dose and age-related outcomes by telomere tagging. This strategy could enable use of TL to assess changes in tolerance of the body to propofol as people get older. However, detection of deletion at the 5 'end or 3 'end of TERC (a ribonucleoprotein that contains the RNA template in telomerase) was not found in our 100 patients, which showed that telomerase in these patients was relatively stable. Hence, we inferred that tolerance of the body to propofol showed strong associations with the inherent genetic factors of aging. TL is a promising predictor of the propofol dose, which is beneficial to individualization of the propofol dose and reduction in the risk of anesthesia.

In addition, we observed that the propofol dose in anesthesia induction was strongly correlated with body weight, sex, and difference of BIS before and after anesthesia induction in 100 participants(Table 2, Fig. 1). Although these data showed that the propofol dose in anesthesia induction is related to many factor, it can be seen that TL contributes the most to the propofol dose by comparing the absolute values of standardized regression coefficients $(0.58,0.21,0.19$, and 0.12$)$ of the four variables. In other words, $T L$ has the greatest influence on the propofol dose in several factors. It may be possible to use telomere length association with external disease factors of aging to determine the dose of propofol in the future, which can greatly reduce perioperative complications.

This was the first study showing a direct association between the tolerance of the body to the propofol dose using TL as a biomarker. TL has been recognized as a strong and informative biomarker of aging. Several studies have shown that TL in PBLs has an inverse association with age and a robust association with mortality risk score[10]. In particular, Dean et al[11] observed a strong relationship between shorter TL and increased overall mortality. TL can be used to predict aging-related health outcomes and is associated with all-cause mortality in humans[10,12]. In the present study, the established links between the propofol dose and TL might (at least in part) testify to an association between the inherent genetic factors of aging and the propofol dose. 
TL has been found to be associated with epigenetic alterations in different populations and environmental exposures. "DNA methylation age" has been used to identify chronological age, and is closely related to telomere attrition[10,13]. Gradual attrition of telomeres occurs during each cell division. The cells become senescent (at least in part), cell-cycle arrest and apoptosis, and cannot divide further when telomeres become very short[14]. Hence, telomeres play a major part in cellular senescence[15] and might contribute significantly to the inherited background of human aging and longevity[16]. Aging results from a dynamic, complex, and multifactorial processes related to a decreased propofol dose by gradual accumulation of different types of cellular and molecular damage[17, 18].TL demonstrated strong associations with propofol dose in our study, and be helpful for determining the propofol dose during aging as well as improving the safety of anesthesia.

Telomere structure is dependent upon genetics, epigenetics, environment and behavioral attitudes, which are present at inconstant extents in different physiological or pathological states[19]. Also, the aging phenotype is closely connected with environmental, genetic, and epigenetic variables[20]. Age-matched elderly people with short telomeres in DNA in PBLs have been shown to have worse survival[21]. Chronological age is a comparatively imprecise measure of an individual's functional and health status. Telomere shortening in one tissue may cause systemic effects[22]. Hence, TL is a predictor of the extent of biological aging and lifespan, or specific for certain biological systems throughout the lifespan. Shortened telomeres are predictive of mortality and associated with mortality across several clinical settings[23, 24]. Despite inconsistent evidence about the degree of heritability[25], TL is considered to be heritable and modifiable by environmental factors. Therefore, TL can represent an individual's biological and functional conditions more precisely than chronological age. Studies have shown that the propofol dose is associated closely with increasing age[3,26]. Furthermore, TL is genetically determined and highly variable: it varies from $35-80 \%$ among individuals and families[27]. TL- and chromosome-specific TL patterns are inherited (e.g., father-to-offspring heritage)[28, 29]. TL and TERC control senescence at the genetic level. Given this information, we can infer that TL of inherent heredity factors can reflect the sensitivity of the body to propofol, and may interact with many other relevant factors.

TL dynamics change constantly over a lifespan, but the rate of telomere change may depend upon genetics, environmental and lifestyle-related factors, stochastic factors, and the genetic mutations of telomerase. Njajou and co-workers ${ }^{30}$ found a significant association between longer TL and better selfrated general health. TL may be an informative biomarker of healthy aging and overall immune competence. Short TL in leukocytes is a cause of impaired immune competence, and has been associated with a higher risk of hospitalization due to infectious disease and infection-related death[31]. Senescence heterogeneity, induced by telomere shortening, depends on the initial variance in $T L[32]$. Hence, $T L$ is a promising predictor of the propofol dose combined with the physiological status of the body.

However, markers of biological aging may change over a lifespan, and a single biomarker may not be sufficient to reflect aging across various biological systems. It is also clear that many mysteries around telomeres and their function remain. In my study, a key question is to what extent the association 
between TL and the propofol dose observed in our study was causal. We did not consider the additional factors, which may have confounded our analyses. Clearly, further studies are needed to evaluate the extent to which TL influences the propofol dose with increasing age.

\section{Conclusion}

We found a positive association between TL in DNA in PBLs and the propofol dose in anesthesia induction. Also, the propofol dose decreased with shorter TL. This strategy could enable use of TL to assess changes in tolerance of the body to propofol as people get older. Hence, TL is a promising predictor of the propofol dose, which is beneficial to individualization of the propofol dose and reduction in the risk of anesthesia.

\section{Abbreviations}

TL: Telomere length; BIS: Bispectral Index; TERC: Telomerase RNA component; PBLs: Peripheral blood leukocytes; qRT-PCR: Quantitative reverse-transcription-polymerase chain reaction

\section{Declarations}

\section{Ethics approval and consent to participate}

This study was approved by Ethics Committee of Guangzhou General Hospital of Guangzhou Military Command and written informed consent was obtained from all participants prior to in the trial. All participants' rights will be protected. The trial was registered prior to patient enrollment at clinicaltrials.gov (NCT03429309, Principal investigator: WeiFeng Tu, Date of registration: February 9, 2018)

\section{Consent for publication}

Authors agree to publish the article.

\section{Availability of data and materials}

All data generated or analysed during this study are included in this published article.

\section{Competing interests}

The authors declare that they have no competing interests. 


\section{Fundings}

This work was financially supported by the Natural Science Foundation of Jiangsu Province of China (Grant Number: SBK2015021666 and LGY2018050).The grant funded the data collection, analysis, and interpretation of data.

\section{Authors' Contribution}

Study design:TWF, ZJL, XY, XCB and YJ. Data collection: XY, XCB, XWB and LZJ. Statistical analysis: XY and XCB. Manuscript preparation: XY. All authors read and approved the final manuscript.

\section{Acknowledgements}

We are thankful for Bo Hu, Jie Peng for providing information for this analysis.

\section{Author details}

${ }^{1}$ Department of Anesthesiology, the Affiliated Lianyungang No. 2 People's Hospital of Jiangsu University, 41 Hailian East Road, Lianyungang, 222006, China.

${ }^{2}$ Department of Anesthesiology, XiAn No. 4 Hospital, XiAn 710004, China

${ }^{3}$ The 74th Group Army Hospital of P.L.A, 20 Eling Southern Road, Huizhou, China

${ }^{4}$ Department of Anesthesiology, The Southern Theater General Hospital, 111 Liuhua Road, Guangzhou, China

\section{References}

1. Anthony M, Paillaud E, Benoît P. Anesthesia in the elderly. Presse Medicale. 2013; 42(2):197-201.

2. Spanjer MRK, Bakker NA, Absalom AR. Pharmacology in the elderly and neweranaesthesia drugs. Best Practice \& Research Clinical Anaesthesiology. 2011; 25(3):355-65.

3. Schnider TW, Minto CF, Shafer SL, et al. The influence of age on propofolpharmacodynamics. Anesthesiology. 1999; 90(6):1502-16. 
4. Blackburn EH, Greider CW, Szostak JW. Telomeres and telomerase: the pathfrom maize, Tetrahymena and yeast to human cancer and aging. Nature Medicine.2006; 12(10):1133-8.

5. Cesare AJ, Reddel RR. Alternative lengthening of telomeres: models, mechanisms and implications. Nature Reviews Genetics. 2010; 11(5):319-30.

6. Capezzone M, Cantara S, Marchisotta S, et al. Short Telomeres, Telomerase Reverse Transcriptase Gene Amplification, and Increased Telomerase Activity in the Blood of Familial Papillary Thyroid Cancer Patients. The Journal of ClinicalEndocrinology \& Metabolism, 2008, 93(10):3950-7.

7. Cawthon RM. Telomere measurement by quantitative PCR. Nucleic Acids Research 2002; 30(10):1-6.

8. Scheinberg P, Cooper JN, Sloand EM, et al. Association of telomere length of peripheral blood leukocytes with hematopoietic relapse, malignant transformation, and survival in severe aplastic anemia. JAMA. 2010; 304(12):1358-64.

9. Du HY, Pumbo E, Ivanovich J, et al. TERC and TERT gene mutations in patients with bone marrow failure and the significance of telomere length measurements. Blood. 2009; 113(2):309-16.

10. Steves CJ, Spector TD, Jackson SHD. Ageing, genes, environment andepigenetics: what twin studies tell us now, and in the future. Age \& Ageing. 2012;41(5):581-6.

11. Calogero C. Biomarkes of aging. Frontiers in Bioscience. 2010; S2(2):392-402.

12. Rode L, Nordestgaard BG, Bojesen SE. Peripheral blood leukocyte telomerelength and mortality among 64,637 individuals from the general population. J NatlCancer Inst. 2015; 107(6):1-8.

13. Zhang $Y$, Wilson $R$, Heiss $J$, et al. DNA methylation signatures in peripheralblood strongly predict allcause mortality. Nature Communications. 2017;8(14617):1-11.

14. de Lange T. How telomeres solve the end-protection problem. Science. 2009;326(5955):948-52.

15. von Zglinicki T. Oxidative stress shortens telomeres. Trends Biochem Sci. 2002;27(7):339-44.

16. Hemann M T, Strong M A, Hao L Y, et al. The shortest telomere, not averagetelomere length, is critical for cell viability and chromosome stability. Cell. 2001;107(1):67-77.

17. Gao X, Zhang Y, Mons U, Brenner H. Leukocyte telomere length and epigenetic-based mortality risk score: associations with all-cause mortality among olderadults. Epigenetics. 2018; 13(8):846-57.

18. Dean S G, Zhang C, Gao J, et al. The association between telomere length andmortality in Bangladesh. Aging. 2017; 9(6):1537-48.

19. Danese E, Lippi G. Telomere length: is the future in our "ends"? Ann Transl Med. 2018; 6(13):280-2.

20. Candore G, Balistreri CR, Listì F, et al. Immunogenetics, gender, and longevity. Ann N Y Acad Sci. 2006; 1089(1):516-37.

21. Cawthon RM, Smith KR, O"Brien E, et al. Association between telomere lengthin blood and mortality in people aged 60 years or older. Lancet. 2003; 361(9355):0-395.

22. Zglinicki T, Martin-Ruiz C, Martin Ruiz. Telomeres as biomarkers for ageingand age-related diseases. Current Molecular Medicine. 2005; 5(2):197-203.

23. Wilbourn RV, Moatt JP, Froy $\mathrm{H}$, et al. The relationship between telomere length and mortality risk in non-model vertebrate systems: A meta-analysis. The Royal Society. 2018; 373(1741):1-9. 
24. Needham BL, Rehkopf D, Adler N, et al. Leukocyte Telomere Length andMortality in the National Health and Nutrition Examination Survey, 1999-2002. Epidemiology. 2015; 26(4):528-35.

25. Prescott J, Kraft P, Chasman DI, et al. Genome-Wide Association Study of Relative Telomere Length. PLoS ONE. 2011; 6(5):1-9.

26. Olmos M, Ballester J A, Vidarte M A, et al. The Combined Effect of Age and Premedication on the Propofol Requirements for Induction by Target-Controlled Infusion. Anesthesia \& Analgesia, 2000, 90(5):1157-61.

27. Codd V, Mangino M, Harst PVD, et al. Common variants near TERC are associated with mean telomere length. Nature Genetics. 2010; 42(3):1-6.

28. Armanios M, Alder JK, Parry EM, et al. Short telomeres are sufficient to cause the degenerative defects associated with aging. Am J Hum Genet. 2009; 85(6):823-32.

29. Nordfjäll K1, Larefalk A, Lindgren $P$, et al. Telomere length and heredity:Indications of paternal inheritance. Proc Natl Acad Sci U S A. 2005;102(45):16374-78.

30. Njajou OT, Hsueh WC, Blackburn EH, et al. Association Between Telomere Length, Specific Causes of Death, and Years of Healthy Life in Health, Aging,and Body Composition, a Population-Based Cohort Study. J Gerontol A Biol Sci Med Sci. 2009; 64A(8):860-4.

31. Helby J, Nordestgaard BG, Benfield T. Shorter leukocyte telomere length is associated with higher risk of infections: a prospective study of 75,309 individuals from the general population. Haematologica. 2017; 102(8):1457-65.

32. Eugene S, Bourgeron T, Xu Z. Effects of initial telomere length distribution onsenescence onset and heterogeneity. J Theor Biol. 2017; 413(15):58-65.

\section{Figures}
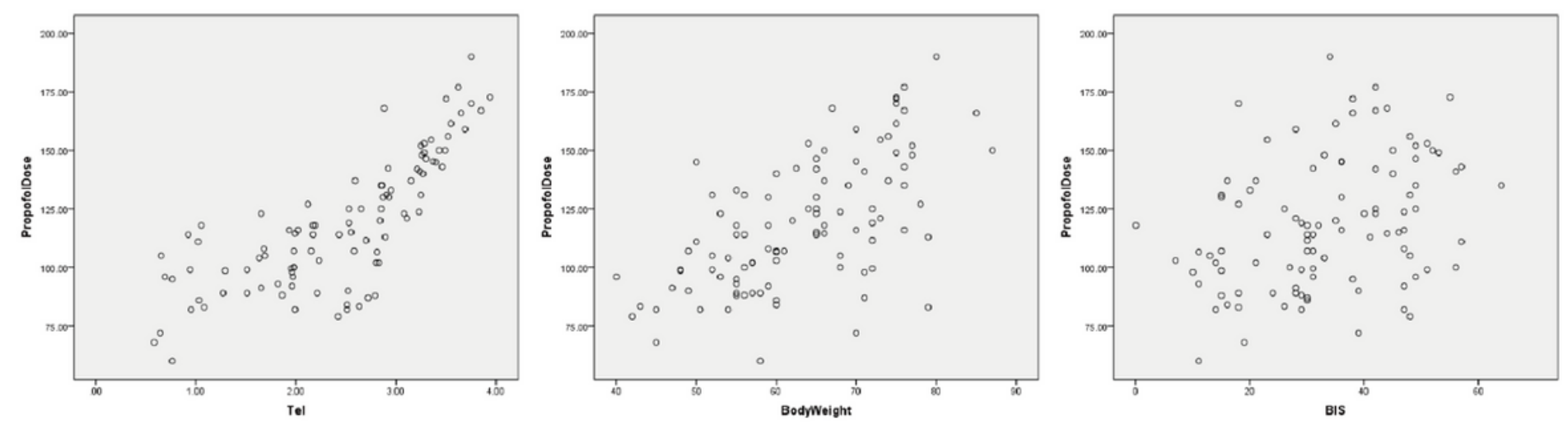

\section{Figure 1}

Linear correlation scatter / dot 


\section{Supplementary Files}

This is a list of supplementary files associated with this preprint. Click to download.

- CONSORT2010checklistofinformation.doc

- CONSORT2010FlowDiagram.doc 